\title{
Inhalt des Jahrgangs 1985
}

Alegsachina, I. V., Autorenbriefe als Quelle für die Bibliographie ,Русские советские писатели. Поәты." . . . . . . . . . . . . . . . . . . . . . . . . . . 436

БАН̈РовА, Н., К сравнительной проблематике в западнославянско甘 антропонимии . 885 Brlentschuow, V., Lev Tolstoj in der Zeitschrift „Die Aktion“ . . . . . . . . . . . . . 670

BחL, I., Zum altsorbischen appellativischen Wortschatz in Toponymen . . . . . . . . . . . 834

Бондарко, А. В., О таксксе . . . . . . . . . . . . . . . . . . . . . . . . . . . 3

Czome, E., Zum Status der charakterisierenden Namen $\quad$. 899

Daneš, F., Zwei Anmerkungen zu den Personalpronomen _ . . . . . . . . . . . . . . . . . . . . $\tilde{\text { s3 }}$

Dew ry, M., Walter Benjamins Interview mit der Zeitung „,Вечерияя Москва“ “ . . . . . 697

Dieckmans, E., Lev Tolstojs Werk in der Kritik der russischen Linken . . . . . . . . . . . . . 664

Dietze, J., Phonetische Slawismen in der Vierten Novgoroder Chronik . . . . . . . . . . . 732

Donnert, E., Schlözer als RuBlandhistoriker . . . . . . . . . . . . . . . . . . . . . . . . 467

DUder, G., R. Trautmann und die klassische russische Literatur. . . . . . . . . . . . . 312

Eckrrt, R., Wolfgang Steinitz und die Vermittlung des Russischen . . . . . . . . . . . 915

Eichler, E., Zum Geleit [H. 3] . . . . . . . . . . . . . . . . . . . . . . . . . . . . . . 305

-, Reinhold Trautmanns Stellung in der deutschen Slawistik . . . . . . . . . . . . . . 306

Gladrow, W., O разных ступенях әнвивалентности между единицами двух яанков . 211

Grochowski, M., Zu syntaktischen, semantischen und pragmatischen Beschreibungsmethoden von Partikeln . . . . . . . . . . . . . . . . . . . . . . . . . . . . . . . 219

GUTSchmid, K., Mitteilungen über den Slawenlehrer Method . . . . . . . . . . . . . 921

Harney, J. - G. SturM, Schlözers Texttreue. . . . . . . . . . . . . . . . . . . . 5iñ

HENGST, K., Sprachkontakt und Entlehnungsprozeß . . . . . . . . . . . . . . . 809

Hösch, E., Schlözer und Finnland . . . . . . . . . . . . . . . . . . . . . . . . . . . 568

Ivanova, K., Diskussion [zu J. Mistrík] . . . . . . . . . . . . . . . . . . . . . . . 52

一, Diskussion [zu D. Stanireva]. . . . . . . . . . . . . . . . . . . . . . . . . . . . 84

_, Pragmatische Beweggründe bei der Wahl einer Genus-verbi-Konstruktion . . . . . . . . 232

Ivić, M., Zu einem Aspekt sprachlicher Interferenz mit einer Exemplifikation aus dem (Ober-)

Sorbischen . . . . . . . . . . . . . . . . . . . . . . . . . . . . 96

Нкус-Борек, Э., Западнославянские ойконимы с мнимым суффиксом -any . . . . . 829

KaČaLA, J., Semantisches und Grammatisches im Satz . . . . . . . . . . . . . . . . . 37

—, Diskussion [zu R. Lötzsch] . . . . . . . . . . . . . . . . . . . . . . . . . . . . . . . . . . 118

Ka EgTner, W., Slaw. *moxidlo in Mecklenburg . . . . . . . . . . . . . . . . . . . . 261

KaIser, E., Appellativische Topolexeme in der dravänopolabischen Toponymie . . . . . . 823

КАлАКуцкАЯ, Л., Грамматические свойства антроПонимии в социолингвистическом аспекте .. . . . . . . . . . . . . . . . . . . . . . . . . . 877

Karolak, St., Von der semantischen zur formalen Struktur des Satzes . . . . . . . . . . 200

KFsikowa, U., Funktionen der Eigennamen in den Romanen von Kornel Makuszyński . . . 904

Климонов, В. Д., Опьт коммуникативно ориентированной модели речепорождения . 237

KörN RR, S., Ortsnamen mit schwierigen Etymologien in der Niederlausitz . . . . . . . . . . 857

KonnaszewskI, M., Die Funktionsweige der Flurnamen im Rahmen der Kommunikationsgemeinschaft des Dorfes . . . . . . . . . . . . . . . . . . . . . . . . . . . . 801

Kordbin, B., Deargumentierung des Agenssubjekts und Sat'senantik in unpersönlichen und Passivkonstruktionen mit dem Element ce des Makedonischen . . . . . . . . . . . . 91 
Laskowski, R., Deverbative Verben der Zustandsveränderung . . . . . . . . . . . . . 42

LaU ER. R., Schlözer und die Grundlegung slavistischer Methodologie . . . . . . . . . . . 634

Lehmaxx, U., Schlözers Ideengut im klassischen Weimar . . . . . . . . . . . . . . . 474

Лихячев, Д. С., Гениальное проиаведение литературы Древней Руси . . . . . . 621

Lötzsch. R., Nichtmorphologische Mittel zum Ausdruck der Passivität im Russischen und Deutschen . . . . . . . . . . . . . . . . . . . . . . . . . 113

-, Agensausdruck und Valenzbeziehungen in passivischen Streckformenkonstruktionen des Russischen und Deutschen . . . . . . . . . . . . . . . . . . . . . . . . 252

Malingoddrs, Pr., Zur frühslawischen Sozialgeschichte im Spiegel der Toponymie . . . . . 873

MARkov, D. F. - G. Zikgenarist, Zum Geleit [H. 4/85] . . . . . . . . . . . . . . . . . 465

Mětšk, F., Zu den Anfängen der Sorabistik im Zeitalter der Spätrenaissance und des frūhen Barock . . . . . . . . . . . . . . . . . . . . . 747

MrChaLk, S., Diskussion [zu M. Ivié] _. . . . . . . . . . . . . . . . . . . . . . . . . . . . . 99

-, Dreisprachigkeit im Rahmen einer grammatischen Konstruktion . . . . . . . . . . . 108

Михневич, А. Е., К характеристике грамматического вааимодействия близкородственньх славянских яакков . . . . . . . . . . . . . . . . . . . . . . . . . . 100

Mistrfk, J., Redundanz der Wortfolge in der Grammatik . . . . . . . . . . . . . . . . . . . . . . 49

MYL'Nikov, A. S., Schlözer am Vorabend seiner Reise nach Rußland (1761) . . . . . . . . 645

Nicolov A, R., Zur Stellung der Pragmatik in der linguistischen Beschreibung . . . . . . . 85

Носов, В., Проблема стиля и жанра в советском литературоведении 60-70-х годов . 701

OHNHEISER, I., Schlözers „Rusgische Sprachlehre“. . . . . . . . . . . . . . . . . . 544

Орел, В. Э., Балканские әтимологии (32-39) . . . . . . . . . . . . . . . . . . 909

Pantentus, M., Būsching und Schlözer. . . . . . . . . . . . . . . . . . . . . . . . 560

Pleskalová, J., Die Kategorie der Possessivität in den Flurnamen Mährens . . . . . . . 865

Ponrt, H., Die frühen baltistischen Arbeiten R. Trautmanns im Spiegel seiner Briefe an

Kazimieras Būga (1921-1924) . . . . . . . . . . . . . . . . . . . . . . . . 350

Popovic, Lj., Konkurrenz von Satz- und Subsatz-(Phrasen-)Konstruktionen . . . . . . . 30

Praż́k, R., Dobrovský und Schlözer . . . . . . . . . . . . . . . . . . . . . . . . 590

Prosst, L., Zur Wiedergabe von Eigennamen beim Utbersetzen vom Deutschen ins Russische 880

$R_{A D E V A}$, V., Wortbildungstendenzen in der bulgariachen Sprache der Gegenwart . . . . . 719

Rosinov, R., Zur Betonung von Nomina und Verben in Petăr Berons „,Рибен буквар““ . . 726

Rơžč̌KA, R., Komplikation und Interaktion in der russischen Grammatik . . . . . . . . 17

Рымут, К., Старые патронимические обрааовавия в славянских яанках . . . . . . 805

Schaller, H., Vergleichende Textpragmatik der slawischen Sprachen . . . . . . . . . . 69

Schmid, H., H. F. Schmids und R. Trautmanns Programmschrift „Wesen und Aufgaben der. deutschen Slavistik" (1927) . . . . . . . . . . . . . . . . . . . . . . 331

SCHRÖT kr, G., 30 Leskien-Briefe aus Jena, Göttingen und Bautzen $(1866-1870)$. . . . . . 361

SChoster-ŚEwc, H , Zur Etymologie einiger mit *z- anlautender slawischer Wörter . . . . 740

-, R. Trautmann und sein Verhältnis zu den Sorben . . . . . . . . . . . . . . . . . 337

-, Zur Geschichte und Etymologie des ethnischen Namens Sorb/Serb/Sarb/Srb . . . . . . 851

Sermase, I., Unveröffentlichte Briefe R. Trautmanns und M. Murkos an den tschechischen Kulturpolitiker Zdeněk Nejedlý . . . . . . . . . . . . . . . . . . . . . . . . . 322

SochaCka, St., Die Rezeption der Arbeiten R. Trautmanns in Polen . . . . . . . . . . . 345

SpRRBer, W., Zur Funktion von Mikrotoponymen im Werke einiger sorbischer Schriftsteller 894

Станишева, Д., Употребление глаголоп движения с учетом фактора адресата в болгарском язике . . . . . . . . . . . . . . . . . . . . . . . . . . . . . . .

Strozyk, G., R. Trautmanns Utbersetzer- und Herausgebertätigkeit zur Propagierung russischer Literatur in den Nachkriegsjahren . . . . . . . . . . . . . . . . 356

Trzun, N. B., Kognitiv-pragmatische Korrelate semantischer Strukturen in einem Zirkelmodell für prozessuelle Sprachanalyse . . . . . . . . . . . . . . . . . . . . . . . 153 
ВАнаГАС, А., Хронологические пластЫ инонавчныХ топонимов ЛитвЫ . . . . . . . 869

Vávas, J., Die Korrespondenz zwischen Dobner und Schlözer über Ursprung und Anfänge

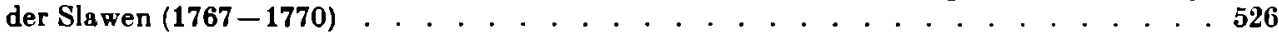

Wargemang, E., Betrachtungen ūber das Reisen in der russischen Literatur des 18. Jahrbunderts .

Wa der, S., Reflexe der drawehnopolabischen Diphthonge in den Namen der Prignitz . . . . 845

Wrazel, W., Die sorbisch-deutschen Siedlungsverhältnisse zwischen mittlerer Elbe und oberer Spree im Spiegel der Personennamen . . . . . . . . . . . . . . . . . . . . 889

Wrtachew, D., Aleko Konstantinors „,Бан̆ Ганю“ . . . . . . . . . . . . . . . . . . 710

Wolle, ST., Schlözer und die jüngere Generation der deutschen Rußlandhistoriker zu Beginn des 19. Jahrhunderts . . . . . . . . . . . . . . . . . . . . . . . 570

ZeIL, L., Die Entwicklung der Slawistik an der Berliner Akademie der Wissenschaften $(1700-1945)$. . . . . . . . . . . . . . . . . . . . . . . . . . 402

ZeIL, W., Die sorbische Sprache und Kultur im Wirken R. Trautmanns . . . . . . . . . 340

Ziramaeist, G., Varnhagen von Ense als Vermittler russischer Literatur im Vormärz (1I) . 119

-, Ungedruckte Briefe von und an Schlözer aus den Jahren 1761-1809 . . . . . . . . . 480

-, Eine unbeachtete Rezension Schlōzers ūber Dobner (1770) . . . . . . . . . . . . . . 539

—, Drei ungedruckte Briefe Schlözers an Gerhard Anton von Halem aus dem Jahre 1802 . . 652

ZIEL, W., Synoptischer Vergleich eines Märchensujets . . . . . . . . . . . . . . . . . 267

Zmmanmann, I., Die Rolle des Lexikons in der Zuordnung von Syntax und Semantik . . . 61

ЗувАРевА, К. А., О некоторых аспектах усвоения творчества М. Е. Салтыкова-Щедрина в Германии на рубеже XIX - XX вв. . . . . . . . . . . . . . . . . . . . 688

\section{REZENSIONEN}

Äquivalezz bei der Translation - besprochen von H. Salevsky . . . . . . . . . . . . . 288 Alexander, M., Der Petraševskij-ProzeB - besprochen von E. Donnert . . . . . . . . . $79 \bar{s}$

Андренчина, К. - С. Влахов - С. ДимитровА - К. Запрянова, Русско-болгарский фразеологическин словарь - besprochen von M. Kовtov . . . . . . . . . . . . . 137

Badmann, W., Die Faszination des Heiligen bei Kliment Ochridski - besprochen von D. Freydank . . . . . . . . . . . . . . . . . . . . . . . . . . . . 611

-, Der Widerspenstigen Zähmung - besprochen von D. Freydank . . . . . . . . . . . . . 760

BAYER, E. - D. ENDLkB, Bulgarische Literatur im Uberblick - besprochen von B. Beyer . 459

Braner ar, E., Slavische Chrestomathie mit Glossaren - besprochen von W. Zeil . . . . . 452

Biedermann, J., Grammatiktheorie und grammatische Deskription in RuBland in der 2. Hälfte des 18. und zu Anfang des 19. Jahrhunderts - besprochen von V. D. Klimonow 936

Bojró, V. - W. Oschires, Lehrbuch der mazedonischen Sprache - besprochen von W. Fiedler . . . . . . . . . . . . . . . . . . . . . . . . . . . . . . . . . . . 789

Brang, P. - M. Zülua, Kommentierte Bibliographie zur Slavischen Soziolinguistik. Bd. I bis III - besprochen von K. Gutschmidt . . . . . . . . . . . . . . . . . . . . . 455

CERMðx, F., Idiomatika a frazeologie ceătiny - besprochen von H. Henschel . . . . . . . 148

DaneŠ, F. - Z. Hlavga a kolektiv, Vötné vzorce v ceătinĕ - besprochen von I. Zimmermann 129

Deppermann, M., Andrej Belyjs ästhetische Theorie des schöpferischen BewuBtseins - besprochen von Ch. Ebert … . . . . . . . . . . . . . . . . . . . . . . . . . 772

Die Hohenfurther Liederhandschrift - besprochen von J. Skutil . . . . . . . . . . . . . 926

Donnebt, E., Das Kiewer Rußland - besprochen von W. Zeil . . . . . . . . . . . . . 617

—, Rußland im Zeitalter der Aufklärung - besprochen von W. Zeil . . . . . . . . . . . 619

Einführung in die multinationale Sowjetliteratur - besprochen von A. Hiersche . . . . . . 767

Encyklopédia slovenských spisovatel'ov - besprochen von L. Richter . . . . . . . . 778

Эстонский фольклор - besprochen von W. Ziel . . . . . . . . . . . . . . . . . . . 938 
ФиРтунатов, Н. М., Творческая лаборатория Л. Толстого - besprochen von G. Dudek . 761

Grimmatik der obersorbischen Schriftsprache der Gegenwart. Morphologie - besprochen von R. Lötzsch . . . . . . . . . . . . . . . . . . . . . . . . . . . . . . . . . . 294

Gictschmid, K. - H. Pört - J. Schultheis, Bibliographie slawistischer Publikationen aus der Deutschen Demokratiechen Republik 1978-1981 - besprochen von W. Zeil . . 757

Hofmasn, T., Das Bauernthema in der sowjetrussischen Prosa der 20er Jahre - besprochen von A. Hiersche . . . . . . . . . . . . . . . . . . . . . . . . . . . . . . . 769

Інформащионный бюллетень МАИРСК, вып. 1-10-besprochen von V. I. Zlydnev . . 299 ІІследования по ксториографии славяноведения и балканистики - besprochen von W. Zeil . . . . . . . . . . . . . . . . . . . . . . . . . . . . . . . . . . 300

Leksikografija i leksikologija - besprochen von B. Müller . . . . . . . . . . . . . . . 133

Јитературный язык XVIII века - besprochen von Ch. Fleckenstein . . . . . . . . . 293

ТукьяновА, Н. А., Лексика совремевньх говоров как объект иаучения - besprochen von K. Gutschmidt . . . . . . . . . . . . . . . . . . . . . . . . . . . . . . 782

Makek, E., Staropolska prozs narracyjns w procesie literackim Rosji wieku XVII i XVIII - besprochen von R. Krawielicki und U. Lehmann . . . . . . . . . . . . . . . . 775

НичевА, К., Българска фразеология - besprochen von M. Kostov . . . . . . . . . . 142

Oвsт, U., Studien zur zweidimensionalen syntagmatiachen Substitution in modernen Prosatexten des Russischen - besprochen von W. Gladrow . . . . . . . . . . . . . . . . 784

Olesce, R., Thessurus linguse dravsenopolabicae. T. I - besprochen von F. Hinze . . . . 930

O Miroslavu Krleži i Augustu Cesarcu - besprochen von M. August . . . . . . . . . . . 779

Памятники общественно-политической мысли в России конда X VII века - besprochen von P. Hoffmann. . . . . . . . . . . . . . . . . . . . . . . . . . . . . . . .616

Павел Наумович Берков (1896-1969) - besprochen von P. Hoffmenn . . . . . . . . 451

Podskalskx, G., Christentum und theologische Literatur in der Kiever Rus' (988-1237) - besprochen von J. Harney - G. Sturm . . . . . . . . . . . . . . . . . . . . .613

Поэтика - besprochen von G. Dudek . . . . . . . . . . . . . . . . . . . . . . . . 764

Polacy w historii i kulturze kraj6w Europy zachodniej - besprochen von D. Scholze . . . 777

Pypin, A. N. - W. D. Spasowirsch, Geschichte der slawischen Literaturen - besprochen von W. Zeil . . . . . . . . . . . . . . . . . . . . . . . . . . . . 456

Rosenkaanz, H., Ortsnamen des Bezirkes Gers - besprochen von K. Müller . . . . . . . 929 Russisches geographisches Namenbuch, Bd. IX -X - besprochen von E. Eichler . . . . . 927

SAfáRIK, P. J., Geschichte der slawischen Sprache und Literatur nach allen Mundarten besprochen von K. Müller . . . . . . . . . . . . . . . . . . . . . . . . . . . . . 790

Schaller, E. W., Die Geschichte der Slavistik in Bayern - besprochen von H. Pohrt . . 793

Schram, G., Eroberer und Eingesessene - besprochen von E. Eichler . . . . . . . . . . . 297

СлАвчевА, Ц., Историческата българистика за чужбина. 1944-1980: Биобиблиографический справочник - besprochen von N. A. Laskeev . . . . . . . . . . . . . . 453

Слинько, А. А., Н. К. Михайловский и русское общественное литературное движение второй половины XIX-начала XX века - beeprochen von D. Jens . . . . . . . . 763

Ślosar, D., Slovotvorný vývoj Ceského slovesa - besprochen von V. Holz . . . . . . . . . . . 934

Словарь-справочник ,Слова о полку Игореве“. Вып. 6 - besprochen von G. Sturm . . 609

Sоснаска, St., Działalność slawistyczna Władysława Nehringa na tle epoki - besprochen von R. Ergetowski . . . . . . . . . . . . . . . . . . . . . . . . . . . . . . . 791

Sprache und Name in Osterreich - besprochen von K. Müller . . . . . . . . . . . . . . 146 
СтаневА, ХР., Струнтура и стилистична функция на сравненията - besprochen von

M. Kostor . . . . . . . . . . . . . . . . . . . . . . . . . . . . . . . . . . 139

Станков, В., Стилистични особености на българския глагол - besprochen von 785

K. Kostov .

Teutscher, Und Reussischer, Dictionarium - besprochen von E. Gūnther. . . . . . . . . 29i)

Творчество писателя и литературвнй продесс - besprochen von K. Kasper . . . . . 774

WaLhafer, C., Maksimilian Volơ̌in als Künstler und Kritiker - besprochen von Ch. Ebert 771

Wege zur Information auf dem Fachgebiet Slawistik - besprochen von G. Sturm . . . . . Tij

\section{TAGUXGSBERICHTE}

Ecrert, R., Colloquium on Early Protestantism and Eastern Europe . . . . . . . . . . 283

-, III. Internationales Symposium zur Phraseologie . . . . . . . . . . . . . . . . . . . . 448

Erchler, E., Siedlungsnamen und Siedlungsformen als Quellen zur Besiedlungsgeschichte Niederö日terreichs . . . . . . . . . . . . . . . . . . . 606

Eichler, E. - K. Gutschmot, Die slawische Namenforschung auf dem XV. Internationalen Kongre B für Namenforschung . . . . . . . . . . . . . . . . . . . . . . . . 801

EndLER, D., IV. Bulgaristische Arbeitstagung . . . . . . . . . . . . . . . . . . . . 755

HiLbert, H.-G., Internationales Michail-Bachtin-Colloquium „Roman und Gesellschaft" . 44:J

Michale, S., Jahrestagung der Kommission für das Studium der grammatischen Struktur der slawischen Sprachen beim Internationalen Slawistenkomitee . . . . . . . . . . .

MülLER, K., Internationales Symposium zu Fragen der etymologischen Forschung, der historischen Lexikologie und Lexikographie . . . . . . . . . . . . . . . . . . . . . . 285

SCHмidt, H., „Die russische Literatur der Aufklärung“ (1650-1825) . . . . . . . . . 602

\section{GEDENKTAGE}

EhEgötz, E. - U. LehManN, Alois Hermann† . . . . . . . . . . . . . . . . . . . 797

EICHLER, E., Josef Filipec 70 Jahre . . . . . . . . . . . . . . . . . . . . . . . . . 940

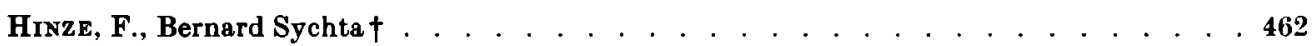

JüGER, G., Rudolf Rủžička zum 65. Geburtstag . . . . . . . . . . . . . . . . . . . . 942

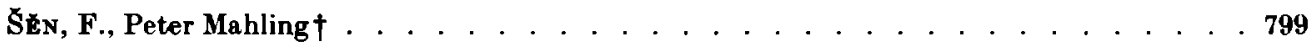

Ziegengeist, G. - E. Diechmand, Georgij Michajlovið̌ Fridlender - 70 Jahre . . . . . . 304

ZIrL, W., In memoriam Paul Nedo . . . . . . . . . . . . . . . . . . . . . . . . . 151

\section{Corrigenda}

S. 69, Anm. 1 : Erscheinungsort und -jahr zum Titel U. Fries ... sind: Wien - Stuttgart 1975.

S. 222, 16. Zeile v. u.: statt zewszad muß zewszqd stehen

S. 222, Satz (17): statt niebawen muß es niebawem heißen

S. 439: Der Vatersname des hier mehrfach genannten Ju. Nechorožev beginnt mit S.

S. 439, Anm. 8: Es muß heißen: 1969, Nr. 10

S. 487, Zeile 3, und S. 539, Zeile 18: Es muß heißen: Titel davon erhalten 


\section{Verzeichnis der Mitarbeiter des Jahrgangs 1985}

Algrsachiva, Irina Vasil'rvna, Kand. der philolog. Wise., Wiss. Mitarbeiterin an der Staatlichen Saltykov-Scedrin-Bibliothek, Leningrad.

Adgost, Moxika, Dipl.-Phil., Wiss. Mitarbeiterin am Zentralinstitut fūr Literaturgeschichte der AdW der DDR, Berlin.

Bayerová, Nadéda a, Dr. phil., Wiss. Oberassistentin an der Pädagogischen Fakultät, Ostrava.

Belestschikow, Valentin, Dr. phil., Wise. Mitarbeiter an der Akademie der Pädagogischen Wissenschaften der DDR, Berlin.

Beyen, Barbara, Dr. phil., Wiss. Mitarbeiterin am Zentralinstitut für Literaturgeschichte der AdW der DDR, Berlin.

Bily, Inae, Dr. phil., Wies. Oberassistentin an der Sektion Theoretische und angewandte Sprachwissenschaft der Karl-Marx-Universität, Leipzig.

Bondanko, Aleksandr Vladmmović, Doktor der philolog. Wiss., Professor am Institut fūr Sprachwissenschaft der AdW der UdSSR, Leningrad.

Crome, Emilia, Dr. phil. habil., Professor em., Loipzig.

Dan rš, Františzr, DrSc., Dozent und leitender Mitarbeiter am Institut für Tschechische Sprache der Tschechoslowakischen AdW, Prag.

Dewky, MichaEL, Dipl.-Phil., Wiss. Mitarbeiter am Zentralinstitut für Literaturgeschichte der AdW der DDR, Berlin.

Dirckmanx, EB ERhazd, Dr. sc. phil., Forschungegruppenleiter am Zentralinstitut für Literaturgeschichte der AdW der DDR, Berlin.

Dietze, Jonchm, Dr. sc. phil., ao. Professor und Direktor der Universitäts- nnd Landesbibliothek Sachsen-Anhalt, Halle.

Donnert, Erich, Dr. sc. phil., o. Professor an der Martin-Luther-Universität Halle-Wittenberg.

Doder, Gramard, Dr. sc. phil., o. Professor an der Karl-Marx-Universität, Leipzig; o. Mitglied der Sëchsischen AdW zu Leipzig.

Emkrt, Christa, Dr. phil., Wiss. Mitarbeiterin am Zentralinetitut für Literaturgeschichte der AdW der DDR, Berlin.

Egkmat, Rann fer, Dr. вc. phil., Professor und Bereichsleiter am Zentralinstitut fūr Sprachwissenschaft der AdW der DDR, Berlin.

Ehzqötz, Erik A, Dr. phil., Wiss. Assistentin an der Sektion Slawistik der Humboldt-Unizersität, Berlin.

Eichrer, Ernst, Dr. sc. phil., o. Professor an der Karl-Marx-Universität, Leipzig; o. Mitglied der Sächsischen AdW zu Leipzig.

Endern, Dietmar, Dr. phil., Hochschuldozent an der Sektion Germanistik und Literaturwissenschaft der Karl-Marx-Universität, Leipzig.

Ergetowski, Ryszard, Doc. Dr. sc., Wise. Mitarbeiter an der Polnischen AdW, Kraków.

Faulstich, Gisela, Dr. phil., Wiss. Oberassistentin an der Sektion Theoretische und angewandte Sprachwissenschaft der Karl-Marx-Univeraität, Leipzig.

Findler, Wilfried, Dr. phil., Forschungsgruppenleiter am Zentralinstitut für Sprachwissenschaft der AdW der DDR, Berlin.

Fleckenstein, Christa, Dr. ac. phil., o. Profeseor und Direktor der Sektion Sprach- und Literaturwissenschaft der Martin-Luther-Universität Halle-Wittenberg.

Fleischer, Holm, Dr. phil., Wiss. Assistent an der Sektion Theoretische und angewandte Sprachwissenschaft der Karl-Marx-Universität, Leipzig.

Fraydank, Dietrich, Dr. phil. habil., ao. Professor an der Martin-Luther-Univergität HalleWittenberg.

Gladrow, Wolfana, Dr. ac. phil., Hochschuldozent an der Sektion Slawistik der HumboldtUniversität, Berlin. 
Grochowski, Macıej, Dr. phil., Wiss. Mitarbeiter am Institut für Polnische Sprache der Polnischen AdW, Warschau.

Günther, Erika, Dr. sc. phil., o. Professor an der Humboldt-Universität, Berlin.

Gutschmid, Kard, Dr. sc. phil., o. Professor und Leiter des Bereichs Sprachwissenschaft an der Sektion Slawistik der Humboldt-Universität, Berlin.

Harngy, JUtta, Dipl.-Phil., Wisg. Mitarbeiterin am Zentralinstitut für Literaturgeschichte der AdW der DDR, Berlin.

Henast, Kare-Heinz, Dr. sc. phil., o. Professor und Leiter der Abteilung Fremdsprachen an der Pädagogischen Hochschule „Ernst Schneller“, Zwickau.

Henscher, Helgonde, Dr. phil., Wiss. Mitarbeiterin am Zentralinstitut fūr Sprachwissenschaft der AdW der DDR, Berlin.

Hirksche, Axton, Dr. phil., Professor am Zentralinstitut für Literaturgeschichte der AdW der DDR, Berlin.

Hinzkrt, Havs-Güster, Dr. phil., Wiss. Assistent an der Sektion Literatur- und Kunstwissenschaft der Friedrich-Schiller-Universität, Jena.

Hinze, Faiedhelar, Dr. phil., Wiss. Mitarbeiter am Zentralinstitut für Sprachwissenschaft der AdW der DDR, Berlin.

Hösch, Edgar, Dr. phil. habil., o. Professor und Vorstand des Instituts für Geschichte Osteuropas und Südosteuropas an der Universität München.

Hofrmans, Petre, Dr. sc. phil,, Wiss. Mitarbeiter am Zentralinstitut für Geschichte der AdW der DDR, Berlin.

Howz, Verena, Dipl.-Phil., Wiss. Assiatentin an der Sektion Slawistik der Humboldt-Universität, Berlin.

How E, Luxz-RArn Er, Dipl.-Phil., Wiss. Mitarbeiter am Zentralinstitut für Sprachwissenschaft der AdW der DDR, Berlin.

Ivanova, Kar.INa, Dr. phil., Dozentin am Institut für Bulgarische Sprache der Bulgarischen AdW, Sofia.

Ivić, Milka, Dr. phil. habil., Professor mit Lehrstuhl für allgemeine Sprachwissenschaft und Serbokroatisch an der Universität Novi Sad.

Jügrr, Gert, Dr. sc. phil., o. Professor und Abteilungsleiter an der Sektion Theoretische und angewandte Sprachwissenschaft der Karl-Marx-Universität, Leipzig.

Jakus-Borkx, Ewa, Dr. phil., Adjunkt an der Pädagogischen Hochschule Opole.

Jena, Detlef, Dr. sc. phil., Hochschuldozent an der Sektion Geschichte der Friedrich-SchillerUniversität, Jena.

KAČaLA, JÁN, DrSc., Direktor des L'udovit-Štúr-Instituts für Sprachwissensehaft der Slowakischen AdW, Bratislava.

Kämmerzähl, Rosemarie, Dr. phil., Wiss. Mitarbeiterin an der Sektion Theoretische und angewandte Sprachwissenschaft der Karl-Marx-Universität, Leipzig.

Ka estner, Walter, Dr. phil., Oberstudienrat a. D., Hamburg.

Kaismr, Elmonore, Dr. phil., Wiss. Rat an der Universität Regensburg.

Kalakdoraja, Larisa, Kand. der philolog. Wiss., Wiss. Mitarbeiterin am Institut für Russische Sprache der AdW der UdSSR, Moskau.

Karolak, Staniseaw, Dr. sc. phil., Professor, Universytet Śląski, Katowice.

Kagper, Karleginz, Dr. phil. habil., o. Professor an der Pädagogischen Hochschule „Clara Zetkin", Leipzig.

Kestrowa, Urszdea, Dr. phil., Adjunkt an der Universität Gdańsk.

Krimonow, Vladmir Dmitrievič, Dr. phil., Wiss. Mitarbeiter am Zentralingtitut für Sprach. wissenschaft der AdW der DDR, Berlin.

Körner, Sigafried, Dr. sc. phil., Fachverantwortlicher für Fremdsprachen im VEB „Otto Grotewohl", Bōhlen.

Kornaszewski, Marek, Dr. phil. habil., Adjunkt an der Universität Poznań.

Kordain, Blagoja, Dr. phil. habil., Professor am Institut für makedonische Sprache der Makedonischen Akademie der W'issenschaften und Künste, Skopje. 
Kostov. KrRr, Dr. phil., Wiss. Mitarbeiter am Zentralinstitut fūr Sprachwissenschaft der AdW der DDR, Berlin.

Kostov, Мантна, Dipl.-Phil., Wiss. Mitarbeiterin am Zentralingtitut fūr Sprachwissenschaft der AdW der DDR, Berlin.

Krawielicki, Renate, Dipl.-Phil., Wiss. Assistentin an der Sektion Slawistik der HumboldtUniversität, Berlin.

Laskeev, Nikolaj Aleksandeovič, Kand. der pädagog. Wiss., Dozent und stellv. Sektorenleiter an der Bibliothek der AdW der UdSSR, Leningrad.

Laskowser, Roman, Dr. phil. habil., Dozent am Institut für polnische Sprache der Polnischen AdW, Krak6w.

La der, Rernand, Dr. phil. habil., o. Professor und Direktor des Seminars für Slavische Philologie der Georg-August-Universität, Göttingen; o. Mitglied der Gōttinger AdW.

Lemmann, Ulf, Dr. phil. habil., o. Professor an der Humboldt-Universität, Berlin.

Lichačrv, Dmitru Sergravič, Dr. phil. habil., Professor am Institut für Russische Literatur (Puškinskij dom) der AdW der UdSSR, Leningrad; o. Mitglied der AdW der UdSSR.

Lötzsch, Rowald, Dr. phil., Wiss. Mitarbeiter am Zentralinstitut fūr Sprachwissenschaft der AdW der DDR, Berlin.

Manngoudis, Phardon, Dr. phil. habil., Professor an der Universität Thessalonike.

Mantey, Johann re, Dr. phil., Wiss. Mitarbeiter am Zentralinstitut fūr Literaturgeschichte der AdW der DDR, Berlin.

Markov, Dmitru Fedorovič, Doktor der philolog. Wiss., Professor und Direktor des Instituts für Slawistik und Balkanistik an der AdW der UdSSR, Moskau; o. Mitglied der AdW der UdSSR.

Meschoang, Marko, Dipl.-Phil., Lehrer im Hochschuldienst an der Sektion Theoretische und angewandte Sprachwissenschaft der Karl-Marz-Universität, Berlin.

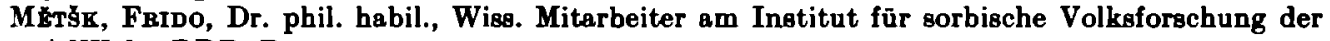
AdW der DDR, Beutzen.

Michalk, Siegrried, Dr. sc. phil., Wiss. Mitarbeiter am Institut fũr sorbische Volksforschung der AdW der DDR, Bautzen.

Michnević, Arnoz'd Efmovič, Doktor der philolog. Wiss., Professor am Jakub-Kolas-Institut fūr Sprachwisenschaft der AdW der BSSR, Minsk.

Mustafk, Jozef, DrSc., Professor und Abteilungsleiter an der J.-A.-Komenskf-Universität, Bratislava.

Müller, Bäreel, Dr. phil., Wise. Mitarbeiterin am Zentralingtitut für Sprachwissenschaft der AdW der DDR, Berlin.

MǗcer, KLA ds, Dr. phil. habil., Wiss. Mitarbeiter am Zentralinstitut für Sprachwisenschaft der AdW der DDR, Berlin.

Myl'nikov, Alegsande Seraegvič, Dr. phil. habil., Professor am Institut fūr Ethnographie der AdW der UdSSR, Leningrad.

Nicolova, Ruseura, Kand. der philolog. Wiss., Dozentin an der Fakultät für Slavische Philologie der Kliment-Ochridski-Universitët, Sofia.

Nolte, Gabriele, Dr. phil., Wiss. Assistentin an der Sektion Theoretische und angewandte Sprachwissenschaft der Karl-Marx-Universität, Leipzig.

Nosov, Vuadmm, Kand. der philolog. Wiss., Dozent am Staatlichen Pädagogischen Institut Kišinev.

Ohnheiser, Ingerorg, Dr. sc. phil., Dozentin an der Sektion Theoretische und angewandte Sprachwissenschaft der Karl-Marx-Universität, Leipzig.

Orel, Vladimme E., Kand. der philolog. Wiss., Wiss. Mitarbeiter am Institut für Slawistik und Balkanistik der AdW der UdSSR, Moskau.

Panteniug, Michael, Dr. phil., Dipl. rer. cult., Journalist, Abteilungsleiter für Kultur bei der Liberal-Demokratischen Zeitung, Halle.

Pleskalová, Jana, PhDr., Wiss. Mitarbeiterin am Institut für tzchechische Sprache der Tschechoslowakischen AdW, Brno. 
Ponqt, Hernz, Dr. phil., Wiss. Mitarbeiter am Zentralinstitut für Literaturgeschichte der AdW der DDR, Berlin.

Popovič, Lu ubomir, Dr. phil. habil., o. Professor an der Universität Belgrad.

Praž́x, Richard, DrSe,, Dozent an der Philosophischen Fakultät der J.-E.-Purkynè-Universiẗ̈t, Brno.

Probst, Lilia, Dr. phil., Lehrerin im Hochschuldienst an der Sektion Slawistik der HumboldtUniversität, Berlin.

Radeva, Vasilka, Kand. der philolog. Wiss., Dozentin an der Kl.-Ochridski-Universität, Sofia.

Richter, Ludwig, Dr. sc. phil., Professor und Forschungsgruppenleiter am Zentralinstitut für Literaturgeschichte der AdW der DDR, Berlin.

Riemschneiden, Romjana, Dipl.-Phil., Lehrerin im Hochschuldienst an der Sektion Theoretische und angewandte Sprachwissenschaft der Karl-Marx-Universität, Leipzig.

Rusinov, Rusin, Dr. phil. habil., Professor an der Universität „Kiril und Metod“, Veliko-Tărnovo.

Rơž̇̌́n, Rudolf, Dr. phil. habil., o. Professor an der Karl-Marx-Universität, Leipzig; o. Mitglied der AdW der DDR, Berlin; o. Mitglied der Sächsischen AdW, Leipzig.

Rymot, Kazrmierz, Dr. phil. habil., Professor am Institut für Polnische Sprache der Polnischen AdW, Kraków.

Salevgky, Heidrmarie, Dr. sc. phil., Hochschuldozentin und stellv. Direktor der Sektion Slawistik an der Humboldt-Universität, Berlin.

Schaller, Helmot, Dr. phil. habil., o. Professor an der Philipps-Universität, Marburg.

Schellen berg, Stefax, Dipl.-Sprachmittler, Wiss.-technischer Assistent am Zentralinstitut für Literaturgeschichte der AdW' der DDR, Berlin.

Schmidt, Honst, Dr. phil. habil., o. Professor an der Martin-Luther-Universität Halle-Wittenberg.

Scholze, Dietrich, Dr. phil., Wiss. Mitarbeiter am Zentralinstitut für Literaturgeschichte der AdW der DDR, Berlin.

Schröter, Geraart, Dr. päd., Lektor an der Sektion Theoretische und angewandte Sprachwissenschaft der Karl-Marx-Universität, Leipzig.

Schuster-Šwc, Heinz, Dr. sc. phil., o. Professor und Leiter des Instituts für Sorabistik an der Karl-Marx-Universität, Leipzig.

Seehase, Ilse, Dr. ac. phil., o. Professor an der Karl-Marx-Universität, Leipzig.

Seifert, Sigafried, Dr. phil., Stellv. Generaldirektor der Nationalen Forschungs- und Gedenkstätten der Klassischen Deutschen Literatur, Weimar.

Šn, Franc, Dr. phil., Wiss. Mitarbeiter am Institut für Sorbische Volksforschung der AdW der DDR, Bautzen.

Skdtil, Jan, PhDr. CSc., Wiss. Mitarbeiter am Mährischen Museum, Brno.

Sochacka, Stamseawa, Dr. phil. habil., Dozentin am Institut Słqgki, Opole.

Sperber, Wolfgang, Dr. sc. phil., ao. Professor an der Karl-Marx-Universität, Leipzig.

Staniševa, Dina, Dr. phil., Dozentin am Lehrstuhl für Slawische Sprachwissenschaft der Kliment Ochridski-Universität, Sofia.

Stern, Annegret, Dipl.-Phil., Wiss. Aspirantin an der Sektion Slawistik der Humboldt-Universität, Berlin.

Strozyr, Gramand, Oberbibliothekar, Stellvertreter des Direktors der Katalogabteilung der Deutschen Staatsbibliothek, Berlin.

Sturm, Gottraied, Dr. phil., Wiss. Mitarbeiter am Zentralinstitut für Literaturgeschichte der AdW der DDR, Berlin.

Thelin, Nirs B., Dr. phil. habil., o. Professor an der Universität Oldenburg.

Vanagas, Alegsampras, Dr. phil., Wiss. Mitarbeiter am Institut fūr Litauische Sprache und Literatur der AdW der Litauischen SSR, Vilnius.

Vívra, Jaroslav, PhDr., Prag.

Waegemans, Emmaxuel, Dr. phil,, Wiss. Assistent an der Katholischen Universität Leuven.

WaUer, Sophie, Dr. phil., Wiss. Mitarbeiterin am Zentralinstitut für Sprachwissenschaft der AdW der DDR, Berlin. 
Weszel, Walter, Dr. sc. phil., Hochschuldozent an der Sektion Theoretische und angewandte Sprachwissenschaft der Karl-Marx-Universität, Leipzig.

Witschew, Donri, Dr. phil., Wiss. Mitarbeiter am Zentralinstitut für Literaturgeschichte der AdW der DDR, Berlin.

Wolle, Stefas, Dr. phil., Wiss. Mitarbeiter am Zentralingtitut für Geschichte der AdW der DDR, Berlin.

Zeil, Liaxe, Dr. phil., Wiss. Mitarbeiterin am Zentralinstitut für Geschichte der AdW der DDR, Berlin.

Zer, Wilhely, Dr. phil., Wiss. Mitarbeiter am Zentralinstitut für Geschichte der AdW der DDR, Berlin.

Ziegengeist, Gerhard, Dr. phil. habil., Profeseor, Chefredakteur der ZfSl und Bereichadirektor am Zentralinatitut für Literaturgeschichte der AdW der DDR, Berlin.

Ziel, WulfhiLd, Dr. phil., Wiss. Mitarbeiterin am Zentralinstitut für Geschichte der AdW der DDR, Berlin.

ZimmermanN, Ilse, Dr. phil., Wisg. Mitarbeiterin am Zentralinstitut für Sprachwissenschaft der AdW der DDR, Berlin.

Zlyonev, Vitalij Ivanovič, Doktor der philolog. Wisg., Professor und Sektorenleiter am Institut für Slawistik und Balkanistik der AdW der UdSSR, Moskan.

Zobareva, Ksenjua Alerservan, Doktor der philolog. Wise., Dozentin am Lehrstuhl für Literatur des Pädagogischen Instituts Omsk. 
Redaktionebol rat: H. H. Blelfeldt (Vorsitzender), W. Beitz, E. Dieckmann, E. Donnert, R. Eckert, E. Eichler D. Preydenk. K. Gable, W. Gladrow, K. Gutschmidt, E. Hexelechneider, A. Hiersche. G. Jager. M. Jahnichen H. Jinger, K. Kasper, E. Kowalski. L. Lehmann, H. Lotzech, P. Nowotny, L. Rlchter, R. Baticke, G. Schlimpert H. Schugter-Sewc, I. Scehage, K.-D. Sthdtke, M. Wegner, G. Zlegengeist, H. Zikmund.

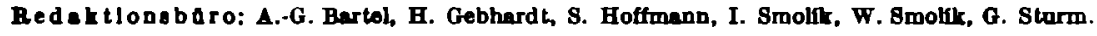

